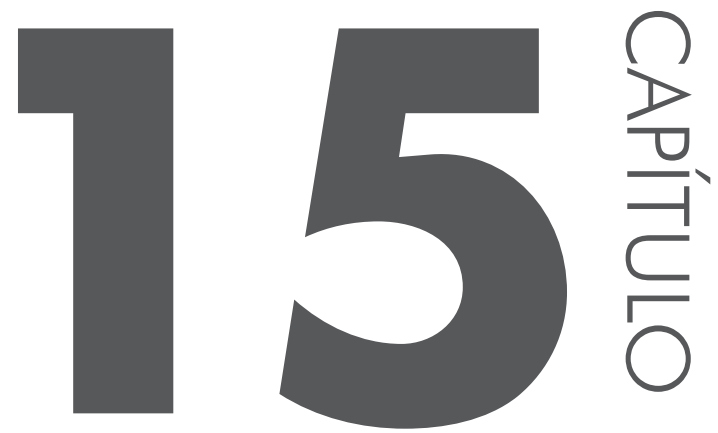

\title{
FLOTAÇÃO DE APATITA UTILIZANDO ÓLEO DE PINHÃO-MANSO
}

Izabela Letícia Almeida de Moraes ${ }^{1}$ André Carlos Silva ${ }^{1}$ Elenice Maria Schons Silva ${ }^{1}$

Agradecimentos: Às agências de fomento CNPq, CAPES e FAPEG pelo auxílio financeiro, primordial para a realização do presente estudo, bem como à Embrapa Agroenergia e à Claraint por ceder amostras utilizadas no estudo e à Universidade Federal de Goiás.

Resumo: As reservas brasileiras de rochas fosfáticas estão concentradas em maioria nos estados de Minas Gerais e Goiás, onde também está concentrada

1 Unidade Acadêmica Especial de Engenharia, Universidade Federal de Goiás - Regional Catalão, Catalão, Brasil.

E-mail de contato: izabela-lets@hotmail.com 
grande parte do cultivo do pinhão-manso (Jatropha Curcas L.), rico em ácidos graxos, que apresenta predominância de ácido linoleico, seguido do ácido oleico e do ácido palmítico. O pinhão-manso é um fruto que contém três castanhas com teor de óleo variando entre $22-48 \%$. Em virtude de sua toxidade, não é uma castanha comestível, o que o torna economicamente viável para ser utilizado em processos industriais. Além da nova exploração do óleo para fins voltados à mineração, o material resultante da prensagem está sendo explorado como biocombustível e tinta para escâner. $\mathrm{O}$ presente artigo apresentou a aplicação do óleo de pinhão-manso (OPM) extraído por prensagem como coletor em testes de microflotação, em virtude da rica composição em ácidos graxos na castanha. $\mathrm{O}$ óleo foi saponificado a quente utilizando álcool $95 \%$ e $\mathrm{NaOH}$ e então foram feitos os testes de microflotação em tubo de Hallimond com o uso de minerais puros apatita com $\mathrm{pH} 8,9$ e 10. Os resultados apresentaram que teve melhor recuperação no pH 10 e para os demais pontos de recuperação para os dois coletores testados apresentaram resultados próximos e superiores a $90 \%$ de recuperação, mostrando que o óleo de pinhão-manso pode ser usado como coletor na flotação deste mineral.

Palavras-chave: Óleo de pinhão-manso. Coletor. Microflotação. Apatita.

Abstract: The Brazilian reserves of phosphate rock are concentrated in majority in the states of Minas Gerais and Goiás, which also concentrate much of the jatropha cultivation (Jatropha Curcas L.), rich in fatty acids and has predominance in linoleic acid, followed by acid oleic and palmitic acid. The Jatropha Curcas is a fruit that contains three chestnuts inside that have oil content ranging from $22-48 \%$. Due to its toxicity, it is not an edible nut which makes it economically viable to be used in industrial processes. Besides the new exploration of oil for purposes aimed at mining, material resulting from the pressing is being explored as biofuel, scanner for paint. This article introduced the application of Jatropha Curcas oil (JCO) extracted by pressing as collector in apatite microflotation tests, due to its rich composition of fatty acids in nuts. The hot oil was saponified using $95 \%$ alcoholic solution and $\mathrm{NAOH}$ and then the microflotation tests were made in Hallimond tube with the use of pure mineral apatite at $\mathrm{pH} \mathrm{8,9}$ and 10 . The results indicate better recovery at $\mathrm{pH} 10$ and other recovery points to the two collectors tested showed similar results and over $90 \%$ recovery indicating that JCO can be used as collector in the flotation of this mineral.

Keywords: Jatropha oil. Collector. Microflotation. Apatite.

\section{INTRODUC̣ÃO}

O aumento do mercado consumidor de fertilizantes fosfatados no Brasil é muito superior ao que se produz. Segundo Fonseca (2013), o governo está exigindo dos grandes grupos investimentos em pesquisas para melhorar a extração 
das reservas brasileiras já descobertas em nome do interesse nacional. As reservas brasileiras de rochas fosfáticas estão concentradas principalmente nos Estados de Minas Gerais com 67,9\% desse total, seguido de Goiás com 13,8\%, São Paulo com $6,1 \%$, que juntos participam com $87,8 \%$ das reservas do país. O estado de Minas Gerais foi responsável, em 2013, por produzir 49\% do fosfato nacional, seguido de Goiás, com 36\%, São Paulo, com 10\%, Bahia, com 4\%, e Tocantins com o restante (NOVAES et al., 2001).

A maioria dos minerais de fósforo das rochas fosfatadas pertence ao grupo da apatita $\left(\mathrm{Ca}_{5}(\mathrm{Cl}, \mathrm{F}, \mathrm{OH})\left(\mathrm{PO}_{4}\right)_{3}\right)$, um fosfato cristalino de cálcio com carbonato e com teor de $\mathrm{P}_{2} \mathrm{O}_{5}$ entre 4 e $15 \%$. Os depósitos de apatita possuem uma complexa mineralogia, contendo impurezas que influenciam na recuperação de fósforo nas usinas de beneficiamento desses minérios. Em virtude disso, a pesquisa apresenta melhorias tecnológicas que já foram realizadas na tentativa de aproveitamento da apatita (SOUZA; FONSECA, 2008).

$\mathrm{O}$ esquema de reagentes de flotação é fator determinante na eficiência do processo. $\mathrm{O}$ uso de reagentes tem por finalidade aumentar as diferenças entre as características originais das superfícies dos minerais presentes no processo. Desta maneira, possibilita-se a separação, uma vez que quanto mais distintas forem as superfícies das espécies a serem separadas, maior a probabilidade de êxito. A flotação deve ser seletiva (BALTAR, 2008).

O potencial dos ácidos graxos como agentes coletores na flotação é reconhecido na literatura. Os coletores aniônicos classificam-se em sulfidrílicos ou oxidrílicos. Dentro do grupo de coletores oxidrílicos destacam-se, dentre outros, os carboxílicos, grupo ao qual pertencem os ácidos graxos (BALTAR, 2008).

Brandão, Caires e Queiroz (1994) realizaram testes de microflotação em tubo de Hallimond com apatita pura, usando como coletores sais de sódio dos ácidos graxos palmítico, esteárico, oleico, linoleico e linolênico em função do pH. Os resultados demonstraram que os ácidos graxos insaturados (linoleico, oleico e linolênico) tiveram desempenho superior em relação aos saturados.

Costa (2012) analisou o uso de óleos vegetais amazônicos na flotação de minérios fosfáticos. Os resultados obtidos no trabalho indicam que é grande a possibilidade de utilização de óleos vegetais amazônicos como coletores na flotação de minérios fosfáticos. O autor destaca que o coletor da semente de maracujá corresponde a uma alternativa aos reagentes atualmente usados na flotação de fosfatos.

O teor de óleo encontrado nas sementes de pinhão-manso varia de 22 a $48 \%$ (BECKER, 2008), porém este teor é em função das condições ambientais e do modo de extração de óleo.

No Brasil, o pinhão-manso ocorre praticamente em todas as regiões do país, sempre de forma dispersa, propagando-se, sobretudo nos estados do Norte e do Nordeste, em Goiás e em Minas Gerais (ALVES, 2010; FREIRE et al., 2009). 
O presente trabalho utilizou o óleo de pinhão-manso, visando buscar uma nova alternativa de reagente coletor na flotação de apatita, analisando o pinhão-manso, uma castanha que é encontra em vários estados do Brasil. A fim de obter matéria-prima de custos menores, as castanhas do pinhão-manso não são comestíveis, o que torna uma alternativa viável do ponto de vista econômico e possui alto teor de ácidos graxos, o que reforça a ideia do seu potencial como coletor.

\section{METODOLOGIA}

O mineral de apatita usado neste trabalho foi adquirido pela Mineração Zé da Estrada, localizada em Araçuaí-MG, e submetido aos procedimentos de moagem, peneiramento e secagem, para sua posterior classificação em faixas granulométricas e armazenamento. O material foi cominuído em moinho de bolas e peneirado para sua posterior separação em faixa granulométrica de 150\#. A etapa de peneiramento foi realizada a úmido. Após o peneiramento, o mineral passou pelo procedimento de filtragem a vácuo para então ser inserido nos recipientes e seguir à etapa de secagem em estufa. As amostras de apatita pura separadas por granulometria foram armazenadas em frascos, prontas para o uso. Uma amostra do mineral passante em 150\# foi submetida à análise química em uma mineradora da cidade de Catalão-GO com o objetivo de identificar os componentes do mineral que foram adquiridos e utilizados nos testes de flotação. O equipamento utilizado para tal análise foi o Espectrômetro de Fluorescência de Raios - X Panalytical, modelo AXIOX MAX série DY n5001.

A caracterização do óleo de pinhão-manso se fez necessária para a interpretação dos resultados de flotação, relacionando as características do óleo que podem influenciar no desempenho do mesmo como coletor. Realizaram-se análises para obtenção do índice de saponificação (IS), grau de saponificação (GS) e índice de acidez (IA), tendo como referência os métodos descritos por Oliveira (2005).

Utilizou-se como coletor o óleo da castanha do pinhão-manso, cedido pela Embrapa Agroenergia localizada em Brasília-DF. As castanhas passaram por um processo de prensagem e filtragem conforme metodologia de Araújo (2012).

Para ser usado como coletor, o óleo de pinhão-manso foi submetido à hidrólise alcalina (saponificação). Optou-se pelo método de saponificação a quente que utiliza solução álcoolica $95 \%$ e $\mathrm{NaOH}$ sob refluxo, pois este requer menor tempo de conversão, já que o álcool proporciona um contato maior do óleo com o $\mathrm{NaOH}$. A eficácia da reação de hidrolise foi verificada visualmente solubilizando o material obtido em água. Se a solubilização fosse completa, considerava-se como saponificado, resultados diferentes eram descartados.

Os testes de microflotação em tubo de Hallimond foram realizados com amostras puras de apatita, numa granulometria de $-100+150 \#(-150+106 \mu \mathrm{m})$. A Tabela 1 apresenta as variáveis operacionais adotadas nos testes. Todos os testes foram 
realizados em triplicata. O condicionamento das amostras foi realizado pelo período de 7 minutos de forma mais concentrada, isto é, colocou-se na parte final do tubo $1 \mathrm{~g}$ do mineral, uma quantidade de coletor que garanta a concentração final desejada $(2,5 ; 5,0 ; 7,5$ ou $10,0 \mathrm{mg} / \mathrm{L})$ e completou-se com água até o limite de $50 \mathrm{~mL}$ de solução para condicionamento. Ao final do condicionamento adicionou-se o restante da água necessária ao procedimento, chegando a uma solução com $320 \mathrm{~mL}$, para então iniciar-se a flotação com intervalo de duração de um minuto.

Tabela 1. Condições dos testes de microflotação de apatita

\begin{tabular}{cc}
\hline Condições & Valores \\
\hline Vazão de ar & $40 \mathrm{~cm}^{3} / \mathrm{min}$ \\
$\mathrm{pH}$ & 8,9 e 10 \\
Faixa granulométrica & $-100+150 \#(-150+106 \mu \mathrm{m})$ \\
Massa do mineral & $1 \mathrm{~g}$ \\
Condicionamento & 7 minutos \\
Flotação & 1 minuto \\
Concentrações & 2,$5 ; 5,0 ; 7,5$ e $10,0 \mathrm{~mL}$ \\
\hline
\end{tabular}

Os ensaios de arraste hidráulico em Tubo de Hallimond revelaram um baixo índice de transporte hidrodinâmico, ou seja, o carreamento de partículas pelo fluxo ascendente gerado com a passagem do ar. Os testes apresentaram um arraste de aproximadamente $0,7 \%$ para a vazão $40 \mathrm{~cm}^{3} / \mathrm{min}$. Dessa maneira, os dados de microflotação serão apresentados desconsiderando valores de arraste. A fim de comparação da performance do óleo de pinhão-manso, utilizou-se o coletor industrial Flotigam 5806, da empresa Clariant.

\section{RESULTADOS E DISCUSSÃO}

A análise de caracterização da amostra de apatita revelou a presença em pequenas quantidades de Barita e Ferro. Entretanto, as concentrações de $\mathrm{P}_{2} \mathrm{O}_{5}$ e $\mathrm{CaO}$ são elevadas, representando $92,54 \%$ de sua composição, condizendo com uma amostra com alto grau de pureza. A Tabela 2 apresenta esses resultados.

Tabela 2. Análise de amostras de apatita após cominuição e classificação - 150\#

\begin{tabular}{ccccccc}
\hline Óxidos & $\mathrm{Nb205}$ & $\mathbf{P}_{2} \mathbf{O}_{5}$ & $\mathrm{Fe}_{2} \mathbf{O}_{3}$ & $\mathrm{SiO}_{2}$ & $\mathrm{Al}_{2} \mathbf{O}_{3}$ & $\mathbf{C a O}$ \\
Composição (\%) & - & 40,50 & 0,07 & 0,94 & 0,06 & 52,04 \\
\hline
\end{tabular}

Fonte: Anglo América (2015). 
O índice de acidez (IA) do óleo de pinhão-manso encontrado foi de 7,67mg KOH/g. De acordo com Berchmans e Hirata (2008) e Canakci e Gerpen (2001), o (IA) na matéria-prima não deve ultrapassar 2,0mg KOH.g-1, ou seja, $1 \%$ p/p. Nesse caso o óleo de pinhão-manso deveria passar por um processo de neutralização para atingir a acidez ideal no caso dos resultados de microflotação derem resultados muito baixo.

O grau de saponificação (GS) apresentou um resultado de 72,75mg de óleo, mostrando que nos testes de microflotação de apatita o (GS) esteve dentro dos parâmetros. Como afirma Oliveira (2005), na prática industrial procura-se trabalhar com um grau de saponificação entre 55 e $75 \%$. Portanto, o grau de saponificação dos testes de microflotação realizados está de acordo com a prática industrial.

O óleo de pinhão-manso apresentou um índice de saponificação (IS) de $89,11 \mathrm{mg} \mathrm{KOH} / \mathrm{g}$, valor baixo de acordo com Tavares (2012), em que seus testes apresentaram um valor médio ente 197,90 e 199,56mg KOH/g óleo. O autor explica que quanto maior o tamanho da cadeia do ácido graxo, menor será o índice de saponificação do óleo, o que pode explicar o (IS) encontrado.

A Figura 1 apresenta a variação da recuperação de apatita com o aumento da concentração dos dois coletores testados em pH 8. Nota-se que em todas as dosagens inferiores a $10 \mathrm{mg} / \mathrm{L}$ o óleo de pinhão-manso teve recuperação próxima e superior a $90 \%$, igualando apenas na concentração de $10 \mathrm{mg} / \mathrm{L}$ ao Flotigam 5806.

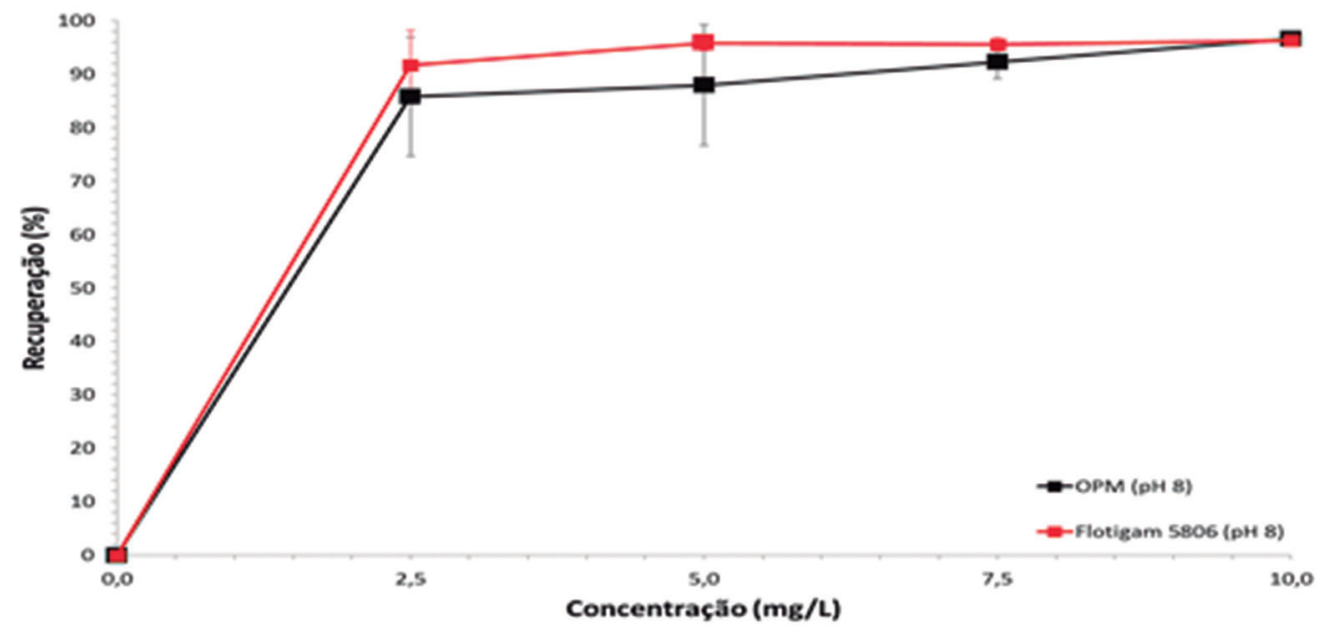

Figura 1. Variação da recuperação de apatita com o aumento da concentração dos coletores (Flotigam 5806 e óleo de pinhão-manso saponificado) em pH 8.

A Figura 2 apresenta a variação da recuperação de apatita com o aumento da concentração dos dois coletores testados em $\mathrm{pH}$ 9. Para a dosagem de 2,5 e $10,0 \mathrm{mg} / \mathrm{L}$ ambos coletores tiveram recuperação semelhante. Contudo, o óleo 
de pinhão-manso obteve recuperação próxima às dosagens de 5,0 e 7,5mg/L do Flotigam 5806.

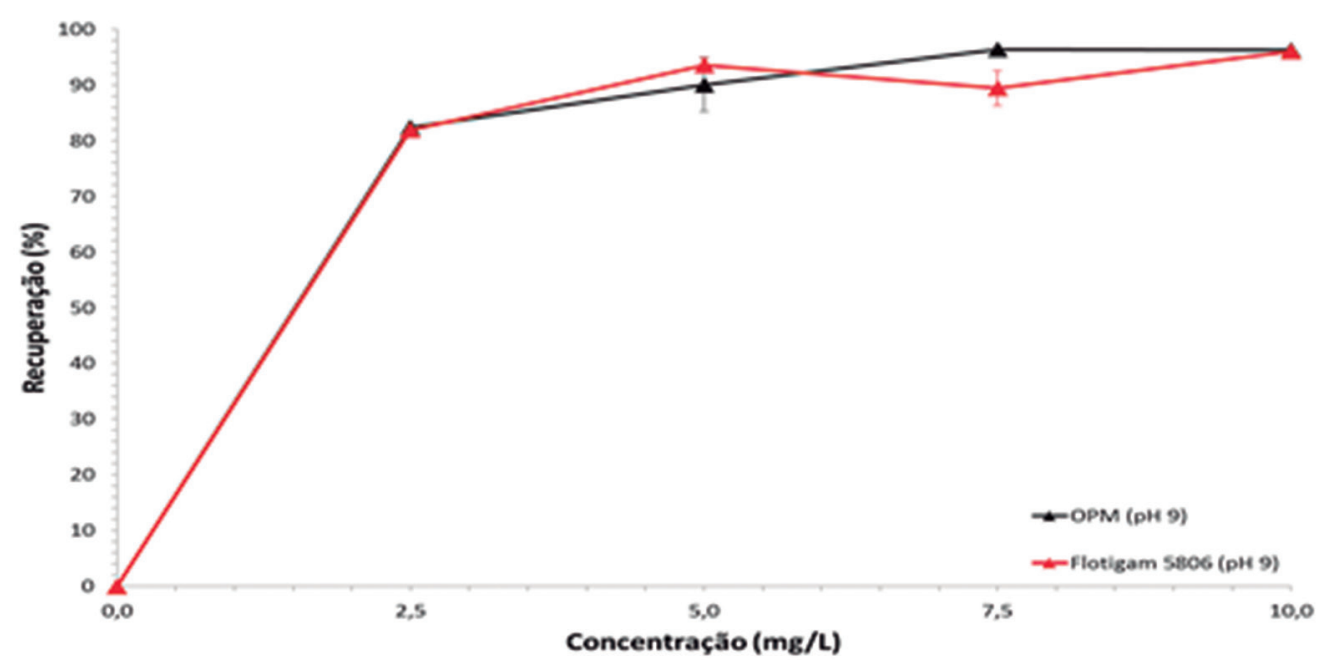

Figura 2. Variação da recuperação de apatita com o aumento da concentração dos coletores (Flotigam 5806 e óleo de pinhão-manso saponificado) em pH 9.

A Figura 3 apresenta a variação da recuperação de apatita com o aumento da concentração dos dois coletores testados em pH 10. O óleo de pinhão teve o mesmo desempenho que o Flotigam 5806 na flotação de apatita. Portanto, é viável a substituição do reagente da Clariant pelo óleo que é, provavelmente, mais barato, além de ser natural.

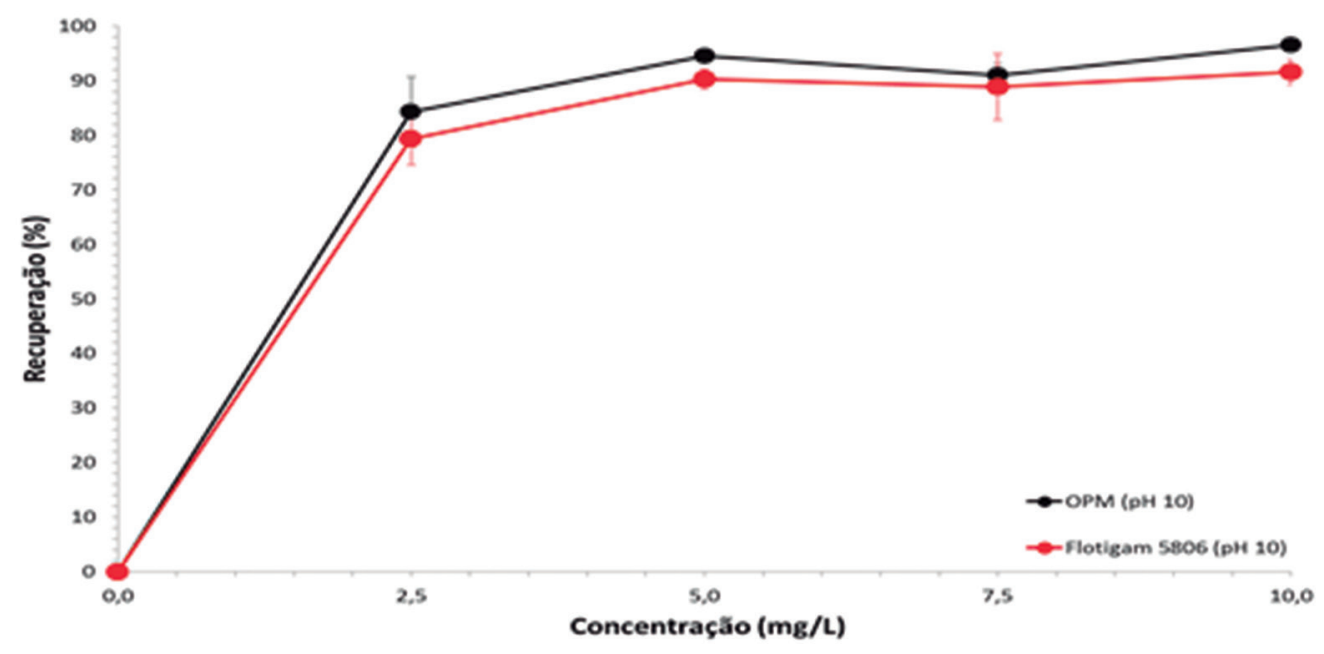

Figura 3. Variação da recuperação de apatita com o aumento da concentração dos coletores (Flotigam 5806 e óleo de pinhão-manso saponificado) em pH 10. 


\section{CONCLUSÃO}

O óleo de pinhão-manso saponificado mostrou-se eficaz como coletor na flotação de apatita em testes de microflotação em tubo de Hallimond. Portanto, o óleo de pinhão-manso apresenta potencial para ser utilizado como coletor industrial, apresentando-se como uma alternativa viável tecnicamente aos reagentes atualmente utilizados na flotação de apatita. A utilização desse reagente coletor alternativo em escala industrial, em substituição parcial ou total aos reagentes atualmente utilizados, seria uma importante contribuição para o setor mineral.

\section{REFERÊNCIAS}

ALVES, M. A. B. Otimização da obtenção de biodiesel etílico de pinhão-manso (Jatropha curcas L.). Dissertação (Mestrado em Agroenergia) - Universidade Federal do Tocantins, Palmas, TO, 2010. 97 p.

ARAÚJO, V. M. Extração de óleo de pinhão-manso para produção de biocombustível. Disponível em: <http://www.producaodebiodiesel.com.br/materias-primas/extracaooleo-pinhao-manso-producao-biocombustivel-biomassa>. Acesso em: 18 fev. 2015.

BALTAR, C. Flotação no tratamento de minério. Recife: UFPE, 2008.

BECKER, K.; MAKKAR, H. P. S. Jatropha curcas: A potential source for tomorrow's oil and biodiesel. Lipid Technology, v. 20, n. 5, 2008.

BERCHMANS, H. J.; HIRATA, S. Biodiesel production from crude Jatropha curcas L. seed oil with a high content of free fatty acids. Bioresource Technology, v. 99, n. 6, p. 1716- 1721, 2008.

BRANDÃO, P. R. G.; CAIRES, L. G.; QUEIROZ, D. S. B. Vegetable lipid oil-based collectors in the flotation of apatite ores. Minerals Eng., v. 7, n. 7, p. 917-925, 1994.

CANAKCI, M.; GERPEN, J. V. The performance and emissions of a diesel engine fueled with biodiesel from yellow grease and soybean oil. Transactions ASAE, v. 46, n. 4, p. 937-944, 2001.

COSTA, D. S. Uso de óleos vegetais amazônicos na flotação de minérios fosfáticos. Tese (Doutorado) - Escola de Engenharia, Universidade Federal de Minas Gerais, Belo Horizonte, 2012. 191 p.

FONSECA, D. S. Sumário mineral - Fosfato. Brasília: DNPM, 2013.

FREIRE, L. M. S.; BICUDO, T. C.; ROSENHAIM, R. Thermal investigation of oil and biodiesel from Jatropha curcas L J. Therm. Anal. Calorim., p. 1029-1033, 2009.

LEMÕES, J. C.; POTES, M. L.; OLIVEIRA, R. J. P.; SILVA, L. L.; SILVA, S. D. A. Determinação do teor de óleo e perfil graxo de acesso de pinhão-manso da Embrapa Clima Temperado. II Congresso Brasileiro de Pesquisas de Pinhão-manso, Brasília, 2011. OLIVEIRA, J. Grau de saponificação de óleos vegetais na flotação seletiva de apatita de minério carbonatítico. Dissertação (Mestrado) - Programa de Pós-Graduação em Engenharia Mineral, Universidade Federal de Ouro Preto, Ouro Preto, 2005. 187 p. 
NOVAES, M.; RAVEN, P. H.; EVERT, R. F.; EICHHORN, S. E. Minerais utilizados para a fabricação de fertilizantes agrícolas. Biologia vegetal. 6. ed. Rio de Janeiro: Guanabara Koogan, 2001.

SOUZA, A. E.; FONSECA, D. S. Fosfato. Brasília: DNPM, 2008.

TAVARES, D. C., Estudo do efeito das misturas de óleos de pinhão-manso, fritura e sebo bovino na produção de biodiesel. Tese (Doutorado) - Instituto de Tecnologia, Departamento de Engenharia Química, Seropédica, Universidade Federal Rural do Rio de Janeiro, Rio de Janeiro, 2012. 
